\title{
Intervention for Severely Obese Children and Adolescents
}

\author{
Kyung Hee Park \\ Department of Family Medicine, Hallym University Sacred Heart Hospital, Hallym University College of \\ Medicine, Anyang, Korea
}

Received January 29, 2019

Reviewed February 13, 2019

Accepted February 19, 2019

${ }^{*}$ Corresponding author

Kyung Hee Park

https://orcid.org/0000-0001-9806-0076

Department of Family Medicine, Hallym University Sacred Heart Hospital, Hallym University College of Medicine, 22 Gwanpyeong-ro 170beon-gil, Dongan-gu, Anyang 14068, Korea

Tel: +82-31-380-3805

Fax: +82-31-380-1782

E-mail: beloved920@gmail.com
Obesity prevalence in children and adolescents is increasing worldwide, and severe obesity prevalence in children and adolescents is also increasing. ${ }^{1}$ According to the student health checkup report, by the Ministry of Education in Korea, severe obesity prevalence increased from $0.8 \%$ in 2008 to $2.0 \%$ in 2017.2

Recently, severe obesity in children and adolescents has been commonly defined as body mass index (BMI) $\geq 120 \%$ of the sexand age-specific 95 th percentile BMI value $(1.2 \times 95$ th percentile). ${ }^{1,3}$ BMI-for-age z-scores have been widely used as the primary outcome to determine intervention effectiveness. However, a recent study has emphasized the limitations of using BMI z-scores as a parameter for intervention effects among children and adolescents with very high BMIs. Instead, they suggested using a relative metric based on the BMI 95th percentile, such as \%BMIp95 and

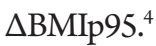

Severe obesity in adolescents is likely to persist into adulthood. A recent study of BMI trajectories from childhood to young adulthood by McGinty et al..$^{5}$ reported more rapid rates of increasing BMI among youth with severe obesity compared with their overweight or nonseverely obese peers. Studies from Korea have reported that the proportion of the population with three or more clustered cardiometabolic risk factors is higher among the severely obese, compared with the proportion among the overweight and the mild-to-moderately obese (30\% vs. $10.9 \%) .{ }^{6}$ Given the extent of previous reports that found an association between severe pediatric obesity and several medical and psychological complications in childhood and adolescence and adulthood ${ }^{3}$, it is imperative to implement effective intervention programs for severely obese children and adolescents in Korea.

In consideration of its complex pathophysiology, multiple intervention components are needed to manage childhood and adolescent obesity. In particular, management of severe obesity requires multidisciplinary weight management services performed by a specialist alongside family- or community-based care. A recently published paper by Christison et al. ${ }^{7}$ in Childhood Obesity, emphasized the value of a medical neighborhood framework with healthcare and community partners for effective coordinated care of children with severe obesity. They also argued that concept-of-care coordination for treating severely obese children will facilitate resource utilization, enhance care capacity, and improve outcomes. To enhance communication between care providers, community sectors, and families, they concluded that interoperable electronic health 
records, a referral and tracking system, and payment for care coordination will play crucial facilitatory roles.

To date, relatively few intervention studies have been conducted for severe pediatric obesity. Moreover, the most common successful approaches for overweight and obese children and adolescents were not sufficiently effective for severe obesity cases. Most intervention programs usually incorporate nutritional education, encouragement of physical activity, and strategies for behavioral modification, including self-monitoring, goal-setting, relapse prevention, stimulus control, and problem solving. Family-based programs or parental involvement and support from peers and one's neighborhood are also important factors for successful weight management. ${ }^{3,7,8}$

Although relatively aggressive interventions, such as inpatient treatment during hospitalization and meal replacement therapy, yielded successful weight loss, patients frequently experienced weight regain after intervention termination. ${ }^{3,9,10}$ Furthermore, inpatient treatment and modest caloric restriction via meal replacement therapy is difficult to maintain for long periods. This suggests a need to develop and implement long-term sustainable intervention programs in the real world, using the chronic care system as a model.

Recently, the results of a 16-week short-term pilot intervention study focusing mainly on severely obese children and adolescents in Korea were published. ${ }^{11}$ In this paper, adding an exercise session to the standard care that included a multidisciplinary program consisting of education and consultation for diet and physical activity, parental education, and behavioral modification was associated with significant improvement in obesity status, body composition, liver function, diastolic blood pressure, and C-reactive protein, compared with baseline. After the intervention, the exercise group showed improvements in body composition, cardiometabolic markers, and lower leg muscle strength, compared with the standard care group. Based on the results of the studies conducted in consideration of the educational and social environment in Korea, establishment of a long-term care plan for severe pediatric obesity in a real-world setting will be needed.

Reviewing the results from intervention programs targeting severe pediatric obesity, severely obese children and adolescents usually remain severely obese or moderately obese, even after achiev- ing a modest reduction in excess body weight through intensive treatment. ${ }^{3}$ These disappointing results can encourage low adherence to intervention programs and high dropout rates. A high dropout rate makes it difficult to accurately evaluate the intervention effect.

However, many intervention studies have not only resulted in improved obesity status, but also in corresponding improvements to body composition, blood pressure, and cardiometabolic biomarkers. In fact, some studies have shown improvements in nonweight-related outcomes only, such as cardiometabolic risk factors, vascular structures or function, and cardiometabolic biomarkers. ${ }^{12,13}$ For example, in the SickKids Team Obesity Management Program, a significant reduction in BMI was not observed, but patients did show improvements in cardiometabolic, psychological, and health-behavior outcomes. ${ }^{14}$

Among the factors related to intervention effects, some studies suggested that age may be an important factor. Knop et al. $.^{15} \mathrm{ob}-$ served that children under 10 years old have shown a larger decrease in BMI z-score compared with adolescents $>10$ years old after a 1-year intervention and a 1-year follow-up period. A 3-year multidisciplinary intervention program by Danielsson et al. ${ }^{9}$ also concluded that young children (aged 6-9 years old) with severe obesity showed improvements in their obesity status, while adolescents (aged 14-16 years old) experienced relatively poor outcomes.

Educating parents or primary caregivers is important for managing childhood and adolescent obesity. Parental and caregiver education should not only focus on physical activity and nutrition, but also on parenting and communication skills, so that they can effectively communicate healthy messages to their child. In fact, given the dependency of the young children on their parents, parental education can be effective for lifestyle improvement, especially in younger children.

Overall, given that many patients maintained a similar degree of obesity after intensive interventions, but experienced improvements in their cardiovascular and metabolic complication profiles, nonweight parameters also need to be focal points. To maximize the effects of lifestyle modification interventions, further studies on physiological, psychological, and environmental factors for severe pediatric obesity and strategies for addressing related factors should be provided. Additionally, observed improvements in cardiometabolic 
risk factors and body weight status need to be better evaluated.

For a feasible, effective, and sustainable long-term intervention program, it is necessary to develop and implement a communitybased intervention model that incorporates the concept of a chronic-care model. To meet this goal, it is essential to utilize the available healthcare resources in the community and stabilize the communication systems between primary and tertiary care through networking within the community.

\section{CONFLICTS OF INTEREST}

The author declares no conflict of interest.

\section{REFERENCES}

1. Skinner AC, Skelton JA. Prevalence and trends in obesity and severe obesity among children in the United States, 1999-2012. JAMA Pediatr 2014;168:561-6.

2. Ministry of Education. Report on student health checkup 2017 [Internet]. Sejong: Ministry of Education; 2018 [cited 2019 Mar 4]. Available from https://www.moe.go.kr/boardCnts/ view.do?boardID $=316 \&$ lev $=0 \&$ statusYN $=\mathrm{W} \& \mathrm{~s}=\mathrm{moe} \& \mathrm{~m}=03$ $02 \&$ жpType $=\mathrm{N} \&$ boardSeq $=73681$

3. Kelly AS, Barlow SE, Rao G, Inge TH, Hayman LL, Steinberger $\mathrm{J}$, et al. Severe obesity in children and adolescents: identification, associated health risks, and treatment approaches: a scientific statement from the American Heart Association. Circulation 2013;128:1689-712.

4. Freedman DS, Butte NF, Taveras EM, Lundeen EA, Blanck HM, Goodman AB, et al. BMI z-scores are a poor indicator of adiposity among 2- to 19-year-olds with very high BMIs, NHANES 1999-2000 to 2013-2014. Obesity (Silver Spring) 2017;25: $739-46$.

5. McGinty SM, Osganian SK, Feldman HA, Milliren CE, Field AE, Richmond TK. BMI trajectories from birth to young adulthood. Obesity (Silver Spring) 2018;26:1043-9.

6. Choi YJ, Lee HS, Jeong WW, Seo YG, Noh HM, Song HJ, et al. Analysis of the associated factors and clinical characteristics of severe obesity in Korean children and adolescents. Korean J
Fam Pract 2018;8:834-40

7. Christison AL, Vaidya S, Tinajero-Deck L, Hampl SE. Application of the medical neighborhood to children with severe obesity. Child Obes 2018;14:461-7.

8. Levine MD, Ringham RM, Kalarchian MA, Wisniewski L, Marcus MD. Is family-based behavioral weight control appropriate for severe pediatric obesity? Int J Eat Disord 2001;30: 318-28.

9. Danielsson P, Kowalski J, Ekblom Ö, Marcus C. Response of severely obese children and adolescents to behavioral treatment. Arch Pediatr Adolesc Med 2012;166:1103-8.

10. Knöpfli BH, Radtke T, Lehmann M, Schätzle B, Eisenblätter J, Gachnang A, et al. Effects of a multidisciplinary inpatient intervention on body composition, aerobic fitness, and quality of life in severely obese girls and boys. J Adolesc Health 2008; 42:119-27.

11. Seo YG, Lim H, Kim Y, Ju YS, Lee HJ, Jang HB, et al. The effect of a multidisciplinary lifestyle intervention on obesity status, body composition, physical fitness, and cardiometabolic risk markers in children and adolescents with obesity. Nutrients 2019;11:E137.

12. Obert P, Gueugnon C, Nottin S, Vinet A, Gayrard S, Rupp T, et al. Impact of diet and exercise training-induced weight loss on myocardial mechanics in severely obese adolescents. Obesity (Silver Spring) 2013;21:2091-8.

13. Many G, Hurtado ME, Tanner C, Houmard J, Gordish-Dressman H, Park JJ, et al. Moderate-intensity aerobic training program improves insulin sensitivity and inflammatory markers in a pilot study of morbidly obese minority teens. Pediatr Exerc Sci 2013;25:12-26.

14. Luca P, Dettmer E, Khoury M, Grewal P, Manlhiot C, McCrindle BW, et al. Adolescents with severe obesity: outcomes of participation in an intensive obesity management programme. Pediatr Obes 2015;10:275-82.

15. Knop C, Singer V, Uysal Y, Schaefer A, Wolters B, Reinehr T. Extremely obese children respond better than extremely obese adolescents to lifestyle interventions. Pediatr Obes 2015;10: 7-14. 